\section{Treatment of Burns}

SIR,-I note that in his article on "The Burnt Patient" (17 August, p. 460) Mr. R. Sanders advocates the repeated dressing of burn wounds; in the emergency procedure he suggests only washing, cooling, and clean dry dressings.

Might I suggest that there is still a place for the old-fashioned remedy of pouring on unrefined or "crude" cod liver oil? This is a naturally sterile preparation and therefore is easy to store and use, but it has the added advantages that a coagulum is produced over the burnt area which is soothing to the burn and which prevents the gross seepage of protein-containing fluid from the burn, thus reducing the common hypoproteinaemic problem in extensive burns but at the same time not forming a completely impermeable eschar as seen with picric acid silver nitrate, etc. The ood liver oil can be poured on repeatedly without removing the previous application and thus distress is avoided and time saved. The only objection to the method is the smell, which is rather pronounced with the crude form, which it is essential to use.-I am, etc.,

GEORGE T. WATTS General Hospital Birmingham

\section{Post-partum Rubella Vaccination, Anti-D Immunoglobulin, and Blood Transfusion}

SIR,-We were interested to read the letters from Messrs. B. Alderman and D. W. Charters (29 June, p. 724) and from Drs. W. M. Edgar and M. H. Hambling (27 July, p. 255) on puerperal rubella vaccination and anti-D immunoglobulin.

Since January 1972 post-partum nubella vaccination has been carried out in Göteborg. In the first year a comparison was made between three commercially available rubella vaccines, Cendevax, Meruvax, and Almevax, considering side effects and seroconversion rates. ${ }^{1}$ Pregnant women have been screened for the presence of haemagglutination-inhibiting (H.I.) antibodies at their first visit to antenatal clinics and those with an H.I. titre $\leqslant 10$ have been offered vaccination, usually within four days after the delivery. Blood samples have been taken immediately before and six to eight weeks after vaccination. To evaluate the influence of anti-D immunoglobulin and blood transfusion on the serological response to rubella vaccination the obstetric reports from 655 rubella-vacoinated women, have been examined; 408 received Almevax, 118 Cendevax, and 129 Meruvax. Eight had received blood transfusions and 58 anti-D immunoglobulin.

The seroconversion rates were $97 \%$ for the controls-that is, the 589 women who had not received transfusion or anti-D prophylaxis-and $100 \%$ for the anti-D group. In the group who had received blood transfusions seroconversions occurred only in four out of eight $(50 \%)$. The difference is statistically significant $(P<0.001)$. The geometric mean titres after vaocination in women showing seroconversion varied from 34 to 38 , but no statistically significant differenoes between the groups were found. One of the women, who had received three units of blood at the delivery, was H.I.negative in the screening test and had an
H.I. titre of 20 after blood transfusion but before vacination. She was again H.I.negative eight weeks after the vaccination. This indicates a possible passive transfer of H.I. antibodies interfering with the serological response to the vaccination. It should be mentioned that the three sera were tested simultaneously and that the H.I. test was repeated three times.

Injection of $20 \mathrm{ml}$ of specific immunoglobulin has been found to reduce the seroconversion rate when given 24 hours after Cendevax vaccination, ${ }^{2}$ Budai et al. ${ }^{3}$ showed that $7.5 \mathrm{ml}$ of $\gamma$-globulin reduced the seroconversion rate when it was given two days before vaccination, while $2.5 \mathrm{ml}$ did not The amount of rubella antibodies in the last dose is probably within the same range as the amount of antibodies in the anti-D immunorglobulin used for prophylaxis.

As shown by the present report anti-D immunoglobulin did not influence the serological response to rubella vaccination. On the other hand blood transfusion reduced the seroconversion rate. It is reasonable to assume that if many units of blood are given a large dose of rubella antibodies can be transferred. We suggest that rubella susceptible women who have received blood a delivery should be vaccinated, but consider it important that the result of the vacination be checked after six to eight weeks. -We are, etc.,

LENA GRILLNER

Department of Virology

Medical Microbiology

LARS FORSSMAN

Department of Obstetrics and Gynaecology,

Eastern Hospital,

1 Grillner, L., et al., Scandinavian fournal of Infectious Diseases, 1973, 5, 237

Whard,, Th., et al., Deutsche medizinische Wochenrift, 1974, 99, 1243

Budai, J., Tóth, M., and Melis, L., Proceedings of the 13th Symposium of the European Asso cation on Poliomyelitis
Helsinki, 1971, p. 210.

\section{The G.P. and the Psychotherapist}

SIR,-In the reorganization of the National Health Service it is proposed that most of the needs of the district shall be met within its own area. It implies a greater degree of co-operation than heretofore between skilled personnel, whether they be general practitioner, social worker, hospital consultant, or nurse, so bringing about greater possibilities not only for the patient but for creating an environment conducive to social well-being and positive health.

In view of these trends and the fact that the psychiatrist specializing in psychotherapy has limited needs for the technical amenities of a hospital, should he not be functioning nearer to the community that he serves? would suggest that the psychotherapist should spend part of his time in a team consisting of a social worker, a psychiatrically trained nurse, and himself, visiting any G.P. in the district who requests his service. The G.P. would gather up patients with whom he needed help from the team, who could use the practice premises outside surgery hours. Hopefully the greater personal contact would make for more effective support and help for the G.P. coping with a psychiatric population variously estimated at between $10 \%$ and $40 \%$ of his practice.

The G.P. has little time to spend with each patient and those with psychological and psychosocial problems are timeconsuming. The visiting psychotherapist would be able to keep the G.P. informed about the latest therapeutic methods and the best use of current psychopharmacological drugs. In his turn he would learn a great deal from the G.P., particularly about environmental problems, social norms, and the difficulties arising in the subcultures which make up that particular district. The idea of a psychiatrist visiting the G.P.'s practice has been not only recommended ${ }^{1}$ but tried successfully on a limited scale, ${ }^{2}$ but not as a team. A nurse, social worker, and psychotherapist should be a useful combination to advise in that twilight area of problems commonly met by the G.P. Such a team might well meet the needs of Dr. M. (22 June, p. 659) and other correspondents who have recently discussed their needs of the acial services in the B.M.F.

The amount of psychological suffering is immense; it has always been with us. However, the man in the street now expects health and happiness, notions engendered by the N.H.S., the media of communication, and advertising. Most psychotherapy is administered either overtly or overtly by persons under one banner or another who are neither medically qualified nor in an allied profession. Much help is offered, some of it excellent, and it has arisen because of the vast need. Surely it is important, therefore, to consider all possible ways of distributing what little professional help there is. Such a scheme is not intended to replace the hospital services but to complement them.

I would be interested in the views of G.P.s and other concerned parties.-I am etc.,

London $\mathrm{W}$

IAN J. MAGDONALD 1 Royal College of General Practitioners, Reports
from General Practice No. 7, 1967.
2 Brook, A., foumal of the College of General
Practitioners, 1967, 13, 127.

\section{Hazard of Propranolol in Treatment of Acute Porphyria}

SIR,-Attention has recently been drawn to the use of propranolol for the symptomatic treatment of the autonomic overactivity of hepatic porphyria in acute relapse. ${ }^{1-5}$ While no controlled studies have been reported, this $\beta$-adrenergic blocker has appeared to be of benefit in certain instances in decreasing the sympathetic nervous system hyperactivity frequently occurring during acute porphyric attacks. Propranolol in lange doses has also been reported to decrease the urinary excretion of $\mathrm{D}$-aminolaevulinic acid (ALA), porphobilinogen, and total porphyrins and to decrease the activity of hepatic ALA synthetase in female rats given the porphyrinogenic compound allylisoprepylacetamide. ${ }^{6}$ We wish briefly to describe an adverse experience with propranolol in the treatment of intermittent porphyria in relapse.

The patient was a desperately ill 33-year-old woman with an acute attack of intermittent porphyria who had a previous history of recurrent porphyria who had a previous history of recurrent urinary tract infection and significant hypertension but without cardiomegaly or congestive heart
failure. Some aspects of her case have previously failure. Some as

Because of severe hypertension, tachycardia, and sweating, unresponsive to oral reserpine, guane- 